\title{
Digital Predistortion of Amplitude Varying Phased Array Utilising Over-the-Air Combining
}

\author{
Nuutti Tervo, Janne Aikio, Tommi Tuovinen, Timo Rahkonen and Aarno Pärssinen \\ Faculty of Information Technology and Electrical Engineering (ITEE), University of Oulu, Finland
}

\begin{abstract}
In this paper, we propose a simple polynomial linearisation technique for nonlinear phased arrays including amplitude control. Due to the large number of antennas and thus power amplifiers in the array, it is inefficient to linearise each power amplifier individually. Therefore, it is demonstrated that the array can be linearised over-the-air using single polynomial. The simulations show that the linearisation is achieved by first linearising the higher driven PAs at the precompression region and then cancelling the compression by the heavily expanding lower driven PAs. The proposed approach offers an alternative way of re-thinking the concept of array linearisation over multiple PAs.
\end{abstract}

Index Terms - digital predistortion, hybrid beamforming, polynomial model, power amplifiers, 5G.

\section{INTRODUCTION}

Large antenna arrays and RF beamforming are becoming common in sub-millimeter and millimeter-wave (mmWave) communications. When increasing the number of antennas and thus power amplifiers (PAs), the power per PA can be decreased. This essentially means that a single PA is contributing less in terms of output power and power consumption. Nevertheless, producing power in sub-mmWave and mmWave regions with high efficiency is a major challenge.

Multiple parallel antennas and PAs enable over-the-air (OTA) combining of signals also for linearisation purposes e.g outphasing [1]. From digital-predistortion (DPD) perspective, OTA-combining is scarely studied. In phased arrays, multiple parallel analog transmitters complicate the DPD because one cannot independently control the input-waveform of individual PAs. In addition, amplitude control is used to reduce the sidelobes and therefore PAs operate at different power levels. In [2] and [3], the array DPD is adressed, but the experimental setup uses a reference antenna in the array far field, which might not be practical approach. Phased array DPD with common feedback is presented in [4] by modelling each PA independently. However, to the best of the author's knowledge, all the previously presented DPD approaches lack the effects of amplitude control and the concept of directed distortion presented in [5]. This paper aims to linearise amplitudecontrolled PA array in the array far field, allowing individual PAs to distort more, but still achieve good linearity in the desired beamforming direction

\section{ARray Modelling}

The basic block chart of the RF beamforming system utilising common DPD over the array is presented in Fig. 1. Amplitude and phase of individual antenna/PA branches are

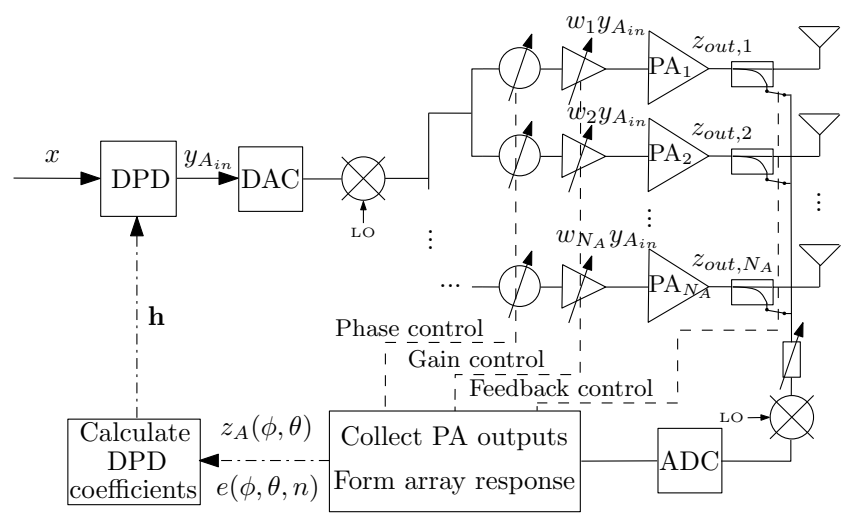

Fig. 1. Block chart of the array DPD process.

controlled to enable beam steering, together with sidelobereduction and interference mitigation. Taylor distribution for 16-element uniform linear array (ULA) achieving $-30 \mathrm{dBc}$ sidelobe level (SLL) is shown in Fig. 2. A single feedback receiver is used for measuring each PA output one-by-one. As a PA model, we use a smoothly compressing memoryless look-up table (LUT) -model, based on the extracted simulation data of $15 \mathrm{GHz} 45 \mathrm{~nm}$ 4-stack CMOS-SOI PA. Amplitude modulation to amplitude modulation (AMAM), amplitude modulation to phase modulation (AMPM) and power-added efficiency (PAE) are exported and the model is presented in Fig. 3. The model is interpolated and extrapolated to cover the instantaneous levels of input waveform.

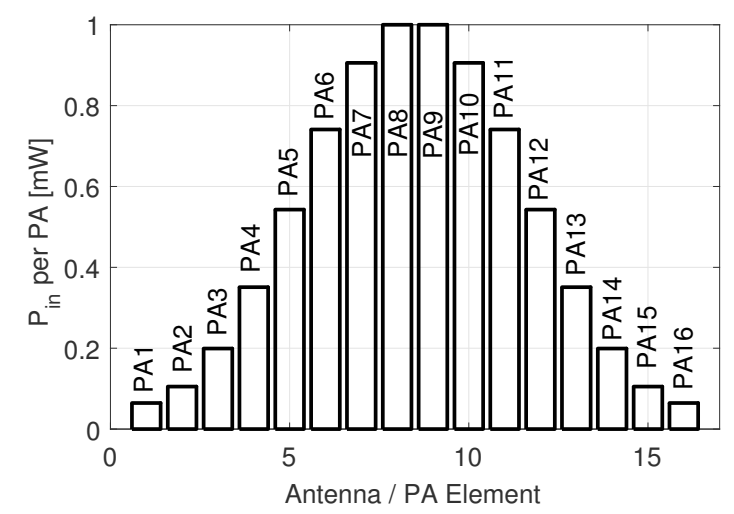

Fig. 2. PA weights for achieving $-30 \mathrm{dBc}$ SLL in linear phased array. 


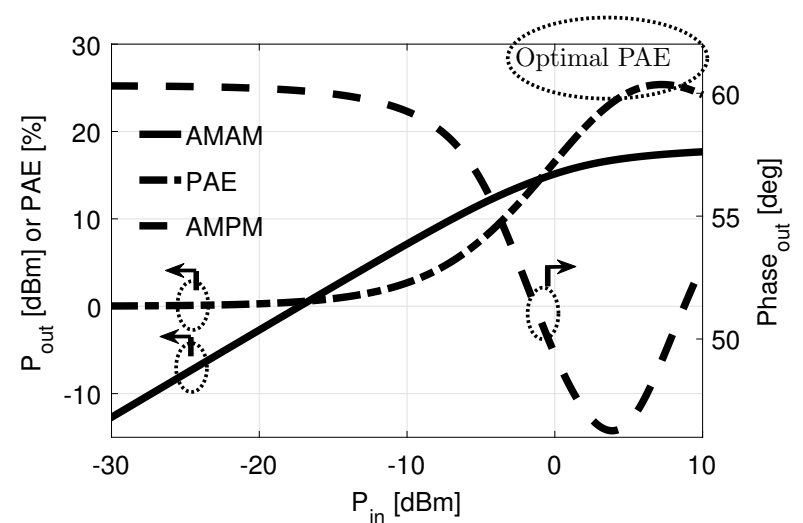

Fig. 3. LUT PA model based on the simulation data of 45-nm 4-stack CMOS PA.

The output of $k$ th PA can be written as

$$
z_{\text {out }, k}=F_{A M}\left(w_{k} x\right) \exp \left(j\left(\arg \left(w_{k} x\right)+F_{P M}\left(w_{k} x\right)\right)\right),
$$

where $x$ denotes the input waveform, $w_{k}$ denotes the beamforming coefficient of $k$ th antenna, $F_{A M}$ denotes the AMAM and $F_{P M}$ the AMPM response of the PA, respectively. The free-space combining can be modelled by array factor [6] for given antenna spacing $d_{A}$ and observation direction $(\phi, \theta)$. If all the antenna elements are identical, the theoretical nonlinear behaviour in the array far field can be expressed as

$$
\begin{aligned}
& z_{A}(\phi, \theta)=\mathbf{F}_{A M}^{T}(\mathbf{w} x) \\
& \exp \left(j\left(\arg (\mathbf{w} x)+\mathbf{F}_{P M}(\mathbf{w} x)+\mathbf{k}^{T} \mathbf{r}\right)\right) F_{S E}(\phi, \theta),
\end{aligned}
$$

where $\mathbf{w}$ denotes the beamforming coefficients, $\mathbf{r}=\left[\mathbf{r}_{x}, \mathbf{r}_{y}, \mathbf{r}_{z}\right]^{T}$ includes the antenna element coordinates in cartesian coordinate system, $\mathbf{k}=$ $\frac{2 \pi}{\lambda}[\sin (\theta) \cos (\phi), \sin (\theta) \sin (\phi), \cos (\theta)]$ denotes the threedimensional wave vector, $F_{S E}(\phi, \theta)$ is the single antenna pattern, and $\lambda$ is the wavelength.

\section{DPD OF RF BEAMFORMING ARRAY}

DPD of the complete array is modelled as

$$
y_{A_{\text {in }}}=\sum_{\substack{l=1 \\ l: \text { odd }}}^{N_{p}} h_{l}^{*}|x|^{l-1} x,
$$

where $h_{l}$ denotes the DPD coefficients and $N_{l}$ is the order of the polynomial. The model can be fitted over several PA responses in least square (LS) sense, as in [4]. Two different LS estimation approaches are compared. The first one is to minimise the sum over LS errors of $N_{A}$ individual PAs as

$$
\min _{\mathbf{h}} \sum_{k=1}^{N_{A}} \sum_{n=1}^{N_{n}}\left|\frac{1}{K_{k}} z_{\text {out }, k}(n)-y_{A_{i n}}(n)\right|^{2},
$$

where $K_{k}$ denotes the linear gain of the $k$ th $\mathrm{PA}, \mathbf{h}=$ $\left[h_{1}, h_{2}, \ldots, h_{N_{l}}\right]^{T}$, and $n$ denotes the time instant over $N_{n}$ time-domain samples [4]. Another approach proposed here is to minimise the array error in the desired direction $\left(\phi_{d}, \theta_{d}\right)$.
By using (2), minimisation of directive LS error can be written as

$$
\min _{\mathbf{h}, \phi=\phi_{d}, \theta=\theta_{d}} \sum_{n=1}^{N_{n}}\left|\frac{1}{K_{\mathrm{A}}} z_{A}(n, \phi, \theta)-y_{A_{i n}}(n)\right|^{2},
$$

where $K_{\mathrm{A}}$ denotes the linear gain of the array in the desired direction, including both the power combining gain and beamforming gain. One should note that in (4) the errors are measured independently while in (5) we are modeling the combined error in the array far field. This is crucial, due to the fact that the errors can add up either constructively or destructively over-the-air. Moreover, it can be proven by Cauchy-Schwarz inequality, that in the desired direction (5) $\leq(4)$. Hence, by allowing the nonlinearities to cancel each other, (5) gives always better or equal performance than (4). For the rest of the paper, DPD with condition (5) is denoted as Array DPD and with condition (4) as Sum DPD.

\section{NumERICAL EXAMPLE}

In system level simulations, the antenna array was horizontally aligned 16-element ULA with a patch antenna and the spacing of $\lambda / 2$ at $15 \mathrm{GHz}$. Taylor amplitude exitation presented in Fig. 2 and $30^{\circ}$ steering direction were assumed,



(a)

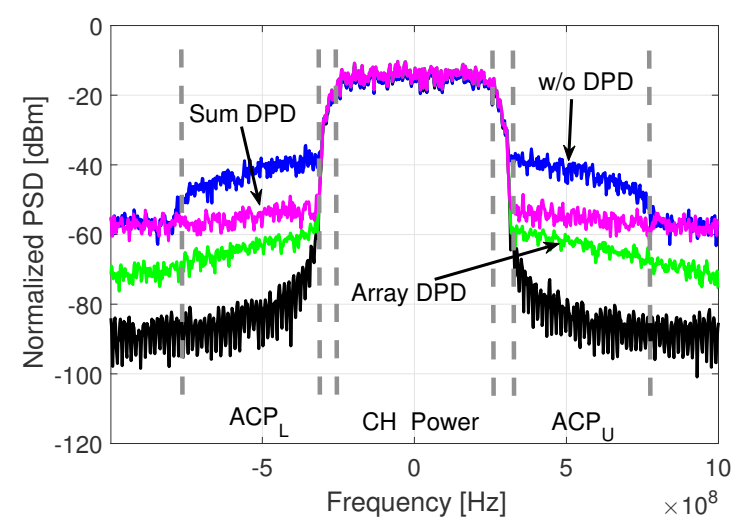

(b)

Fig. 4. Observed spectrums in (a) individual PA outputs and in (b) the array far field in the desired direction with and without DPDs. 


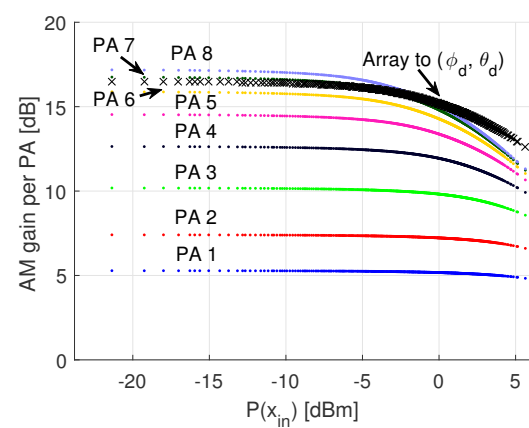

(a) AMAM w/o DPD

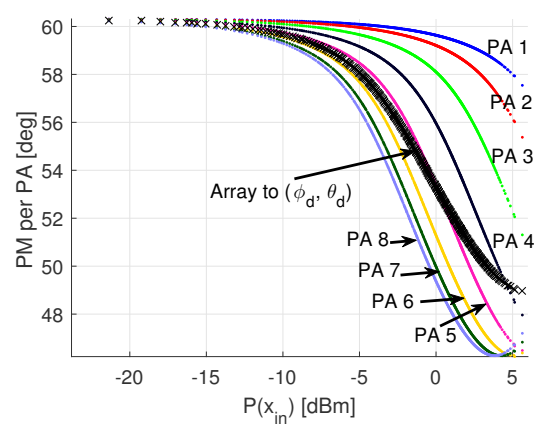

(d) AMPM w/o DPD

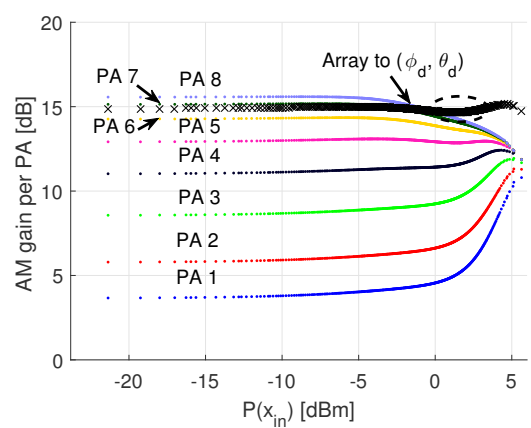

(b) AMAM w Sum DPD

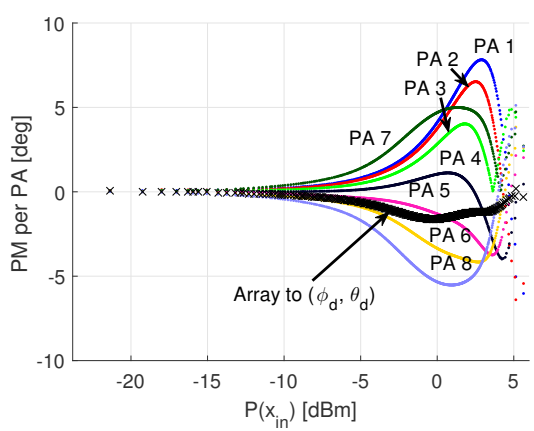

(e) AMPM w Sum DPD

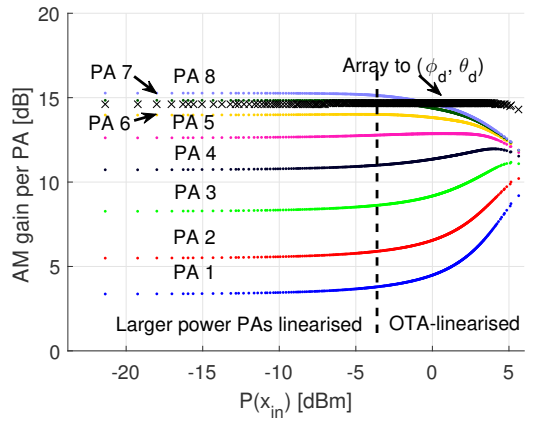

(c) AMAM w Array DPD



(f) AMPM w Array DPD

Fig. 5. AMAM and AMPM relative to common input signal without DPD and with two different DPD methods.

and each PA followed the model presented in Fig. 3. Input waveform was $500 \mathrm{MHz}$ wide 256-QAM modulated signal with raised cosine pulse-shaping, and 4x oversampling were assumed from the feedback receiver. The DPD training was done over 1024 symbols utilising LS estimation.

The power spectral densities (PSDs) at each PA output and the combined PSDs in the desired direction with and without DPDs are presented in Fig. 4. Both DPD methods make smaller power PAs more nonlinear, but the overall response is linearised in the desired direction compared with the case w/o DPD. Furthermore, it is observed that Array DPD outperforms the Sum DPD in the array far field.

To explain the behaviour, the AMAM and AMPM of 8/16 PAs and the normalised array output with given beamformer and DPDs are presented in Fig. 5. The rest of the PA weights are symmetrical with the first eight. Figs. 5a and d show that the PAs have different nonlinear curves, but the shape of the array AMAM and AMPM is smooth in the desired direction. This indicates that the array can be modelled using single polynomial. In Figs. 5b and e, the DPD is calculated based on the condition (4). Because the errors of individual PAs are weighted as powers, the Sum DPD linearises mostly the PAs with higher power levels. In Sum DPD, the expansion of lower driven PAs increases the total error, decreasing the linearity in higher power levels. Figs. $5 \mathrm{c}$ and $\mathrm{f}$ presents the DPD with condition (5). The Array DPD linearises the array by two main mechanisms. In weakly compressing region, the DPD linearises the response of higher driven PAs. For larger signal levels, the DPD makes lower driven PAs to expand for canceling the compression of higher driven PAs (denoted as OTA-combining in Figs. 5c and f). Because of the power difference, more PAs (10/16) are expanding than compressing (6/16). As expected, Array DPD gives better linearity than Sum DPD.

In the simulations, it was noted that the nonlinearity changes the original amplitude tapering which was presented in Fig. 2. This will have significant effect for the sidelobes of the array beam. Fig. 6 shows the rms power weights of each antenna element in the phased array. The PAs are operating in different power levels which affects to the instantaneous levels of the waveform in each individual antenna input. For the higher driven PAs, the rms power is reduced compared to the PAs driven with lower signal levels. Even though both DPD methods expands the lower driven PAs, they are not significantly changing the rms powers.

Fig. 7 presents the beam pattern in the array far field over the azimuth half plane. DPDs do not affect significantly to the shape of the main lobe and hence only one channel power beam is presented. As expected based on Fig. 6, the SLL is increased to $-20 \mathrm{dBc}$ as it was $-30 \mathrm{dBc}$ with linear PA array. For illustrating the nonlinear behaviour of the array, adjacent channel powers (ACPs) in the array far field are calculated. The presented ACP is the maximum between lower $\left(\mathrm{ACP}_{\mathrm{L}}\right)$ and upper $\left(\mathrm{ACP}_{\mathrm{U}}\right)$ in each spatial direction. ACPs are observed to be direction dependent, especially if any amplitude variations between the PA branches are present. Both DPD 


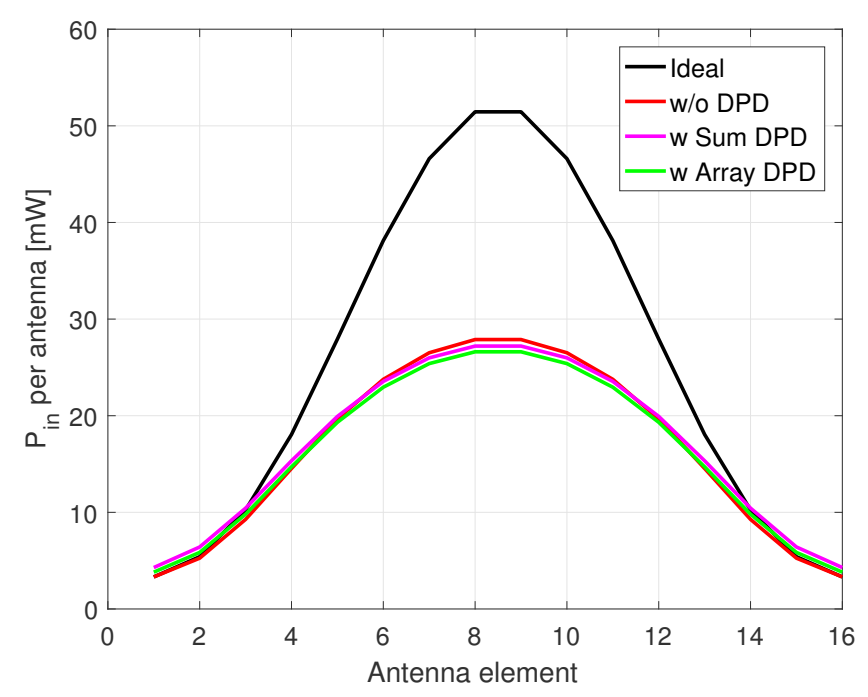

Fig. 6. Antenna weights for ideal linear PA array, and for nonlinear PA array with and without DPDs.

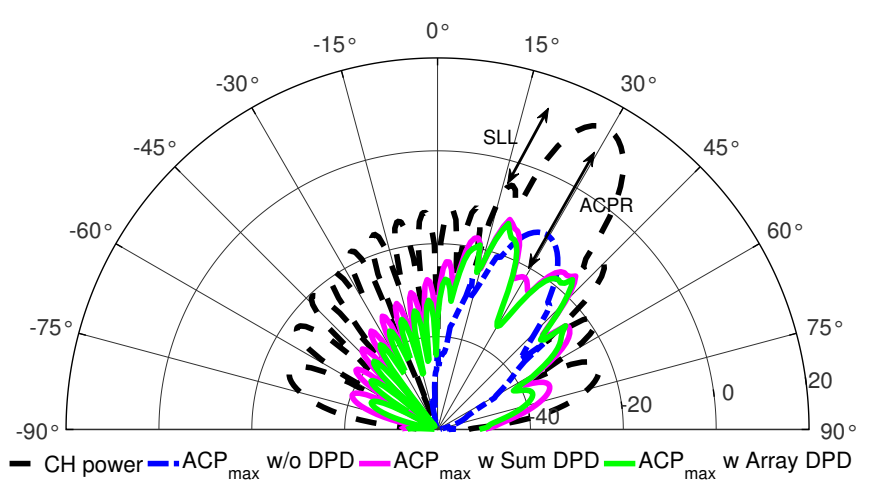

Fig. 7. Channel power and $\max \left(\mathrm{ACP}_{\mathrm{L}}, \mathrm{ACP}_{\mathrm{U}}\right)$ in the array far field.

methods improve the ACP in the desired direction while they are not having impact to beamforming itself. Because the Array DPD takes the beamforming direction into account, it outperforms the Sum DPD.

In practice, the array DPD training should be adaptive also for different beamforming coefficients. In Fig. 8, the DPD is trained to $30^{\circ}$ angle, and the steering angle of the array is varied $\pm 10^{\circ}$ away from the training direction. It can be seen that Array DPD is beneficial when the steering angle is around $\pm 5^{\circ}$ different than the DPD training angle. On the contrary, Sum DPD has almost constant ACP over the simulated steering angles, indicating that it is more robust for adaptive beam steering.

\section{CONCLUSION}

We proposed a DPD technique for linearising the RF beamforming array with common DPD in a certain spatial direction. In mathematical analysis, it was concluded that the nonlinear behaviour should be modelled in the array far field instead of only at each PA output. The proposed DPD linearises the

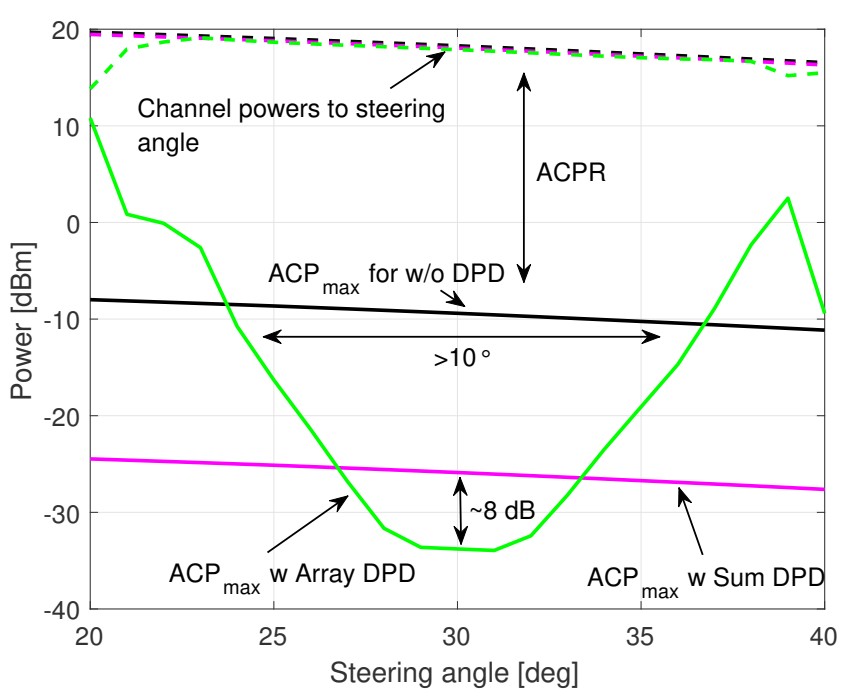

Fig. 8. DPD sensitivity for varying steering angle.

array in two main mechanisms. In lower signal levels, the DPD is linearising mainly the higher driven PAs. At higher signal levels, the lower driven PAs are heavily expanding which cancels some of the compression of higher driven PAs. The proposed DPD minimises the ACP in the beamforming direction while it has no impact on the main lobe power.

The practical array DPD must not only tolerate the beamforming, but also standard phenomena such as memory effects, adaptive load modulation etc. must be addressed with more complex models. However, many of these problems are rather well studied and do not depend on the array behaviour. In order to apply the presented approach in practice, major effort must be addressed in the practical feedback strategy and adaptive DPD training.

\section{ACKNOWLEDGMENT}

This research has been supported by Infotech Oulu Doctoral Programme, Finnish Funding Agency for Technology and Innovation (Tekes), Nokia Oyj, Esju Oy and CoreHW Oy.

\section{REFERENCES}

[1] C. Liang and B. Razavi. "Transmitter Linearization by Beamforming", in IEEE Journal of Solid-State Circuits, vol. 46, no. 9, pp. 1956-1969, Sept. 2011.

[2] P. Y. Wu, Y. Liu, B. Hanafi, H. Dabag, P. Asbeck and J. Buckwalter. "A 45-GHz Si/SiGe 256-QAM Transmitter with Digital Predistortion”, IEEE MTT-S Int. Microw. Symp. Dig., Phoenix, AZ, 2015, pp. 1-3.

[3] H. T. Dabag, B. Hanafi, O. D. Grbz, G. M. Rebeiz, J. F. Buckwalter and P. M. Asbeck, "Transmission of Signals With Complex Constellations Using Millimeter-Wave Spatially Power-Combined CMOS Power Amplifiers and Digital Predistortion", in IEEE Trans. Microw. Theory Tech., vol. 63, no. 7, pp. 2364-2374, July 2015.

[4] S. Lee, M. Kim, Y. Sirl, E.R. Jeong, S. Hong, S. Kim and Y.H Lee. "Digital Predistortion for Power Amplifiers in Hybrid MIMO Systems with Antenna Subarrays," in IEEE 81 st Vehicular Technology Conference (VTC Spring), 11-14 May 2015, pp.1-5

[5] N. Tervo, J. Aikio, T. Tuovinen, T. Rahkonen and A. Paerssinen. "Effects of PA Nonlinearity and Dynamic Range in Spatially Multiplexed Precoded MIMO Systems," in 22th European Wireless Conference, Oulu, Finland, 2016, pp. 1-6.

[6] C. Balanis. Antenna Theory: Analysis and Design, Wiley, 2012 